\title{
Moving dark energy and the CMB dipole
}

\author{
A. L. Maroto \\ Departamento de Física Teórica, Universidad Complutense de Madrid, 28040 Madrid, \\ Spain \\ E-mail: maroto@fis.ucm.es
}

\begin{abstract}
We explore the possibility that the rest frames of CMB, matter and dark energy differ one from another, i.e. they do not converge on very large scales. In such a case, the usual interpretation of the CMB dipole as being due to the relative motion of the observer with respect to the CMB rest frame is not appropriate. Instead, we find that the measured dipole is due to the observer motion relative to the cosmic center of mass rest frame. This means, in particular, that even an observer at rest with respect to the $\mathrm{CMB}$ radiation could measure a non-vanishing dipole anisotropy, provided dark energy is moving with respect to the CMB. We also consider the consequences of moving dark energy for the determination of cosmic bulk flows.
\end{abstract}




\section{Introduction}

Standard cosmology assumes homogeneity and isotropy of the Universe on very large scales. The presence of density perturbations implies that when averaged over small volumes, matter can have a non-vanishing streaming velocity with respect to the $\mathrm{CMB}$ radiation, the amplitude of such motions depending on the actual power spectrum of density fluctuations. However, as we take larger and larger averaging volumes, convergence of both reference frames is expected according to the Cosmological Principle. In other words, matter and radiation should share a common rest frame. However, the observational situation is far from clear. Recent large-scale peculiar velocity surveys have measured the dipole of the peculiar velocity field on different scales, trying to determine the volume size at which the streaming motion vanishes. Although there is evidence of convergence on very large scales $\gtrsim 100 h^{-1} \mathrm{Mpc}$ in some works [1, non-vanishing bulk flows with amplitudes $\gtrsim 600 \mathrm{~km} \mathrm{~s}^{-1}$ with respect to the CMB have also been measured in other surveys on those distance scales [2, 3, 4, although the results do not agree in the direction of the motion, and they have been argued to be affected by systematics [5]. The possibility that motions with such a large amplitude could be accomodated within the standard model of structure formation was studied in [6] and more recently in [5].

On the other hand, the dominant contribution to the CMB dipole anisotropy is usually attributed to the Doppler effect due to the observer motion with respect to the last scattering surface [7, 8, 9]. Substracting the contribution from the solar system motion relative to the Milky Way, and the Milky Way relative to the Local Group (LG) [10], the measurement of the CMB dipole has been used to obtain the relative velocity of the LG with respect to the CMB, which according to COBE [9] is $627 \pm 22 \mathrm{~km}$ $\mathrm{s}^{-1}$, towards Galactic coordinates $l=276 \pm 3^{\circ}, b=30 \pm 3^{\circ}$. This result seems to be compatible with the direct determination of the LG velocity with respect to the rest frame defined by certain SNIa host galaxies [11. It is however inconsistent with the result in [2], according to which the LG is moving at $561 \pm 284 \mathrm{~km} \mathrm{~s}^{-1}$ towards $l=220^{\circ}$, $b=-28^{\circ}$ with total angular error of $\pm 27^{\circ}$, with respect to the frame defined by the 119 Abell clusters contained within a $150 h^{-1} \mathrm{Mpc}$ distance (see also [3]).

Some alternatives to the standard $\Lambda$ CDM cosmology suggest different explanations for those discrepancies. Thus for instance, according to [9], the older proposal of [12] in which matter and $\mathrm{CMB}$ velocities could have started to differ after decoupling, predicts the existence of bulk motions over horizon scales. Other hypothesis is that the dipole is not kinematic, but of cosmological origin, due to an entropy gradient (isocurvature perturbation) on super-Hubble scales [13, 14 or to preinflationary remnants in the density perturbations [15. In any case, according to the previous discussion, the possibility that matter and radiation have different rest frames is not observationally excluded.

However matter and radiation are not the dominant components of the universe today. Recent observations of SNIa [16] combined with CMB anisotropies [17] suggest 
that the rate of expansion of the universe is increasing, the acceleration being driven by an unknown form of dark energy with a relative density $\Omega_{\Lambda}=0.73 \pm 0.04$ and equation of state $w_{\Lambda}<-0.78$ (at the $95 \%$ c.l.). (For the more recent three-year WMAP data 18 ] $\Omega_{\Lambda}=0.72 \pm 0.04$ and $w_{\Lambda}=-0.97_{-0.09}^{+0.07}$ (WMAP+SNLS) for a flat universe). Although the nature of dark energy is a complete mistery, several models have been proposed in which dark energy appears either as a pure cosmological constant term in the Einstein equations; as a perfect fluid with appropriate equation of state; as an extremely light scalar field running down the slope of a given potential in the quintessence models [19]; or as a scalar field with non-canonical kinetic term (k-essence) [20]. In all those cases, dark energy is completely decoupled from matter and radiation, its only effects being of gravitational nature (see [21] and references therein).

The existence of a common rest frame is expected for strongly coupled fluids, as is indeed the case for baryonic matter and radiation before recombination. However, this might not be true at the present epoch when matter, radiation, and presumably dark energy are almost completely decoupled. In such a case, it makes sense to explore the possibility that the different components have different rest frames.

\section{Velocity perturbations}

Let us therefore consider a cosmological scenario with three perfect fluids: radiation, matter and dark energy, whose equations of state read $p_{\alpha}=w_{\alpha} \rho_{\alpha}$ with $\alpha=R, M, \Lambda$. For the sake of generality, we will allow the dark energy equation of state to have a smooth dependence on redshift $w_{\Lambda}(z)$. The energy-momentum tensor of each fluid will take the form:

$$
\left(T_{\nu}^{\mu}\right)_{\alpha}=\left(\rho_{\alpha}+p_{\alpha}\right) u_{\alpha}^{\mu} u_{\nu \alpha}-p_{\alpha} \delta_{\nu}^{\mu}
$$

Since in this work we are only interested in the effects of fluids motion on the CMB dipole, it is sufficient to take into account only the evolution of velocity perturbations, i.e. we will not consider density or pressure perturbations. In addition, since we will only concentrate on the dipole anisotropy, it is enough to consider the homogeneous part of the velocity fields. The presence of inhomogeneities will contribute to higher multipoles, (see for instance [22]). Therefore, for this particular problem we can write:

$$
\begin{aligned}
& \rho_{\alpha}=\rho_{\alpha}(\eta), \\
& p_{\alpha}=p_{\alpha}(\eta), \\
& u_{\alpha}^{\mu}=\frac{1}{a}\left(1, v_{\alpha}^{i}(\eta)\right)
\end{aligned}
$$

where $\eta$ is the conformal time. The effects of dark energy perturbations on higher multipoles have been considered in 23 , mainly in connection with the problem of the low CMB quadrupole.

In the following we will assume that $\vec{v}_{\alpha}^{2} \ll 1$ and we will work at first order in perturbation theory. Since the fluids only support velocity perturbations, the form of 
the space-time metric will be given by the most general vector-perturbed FriedmannRobertson-Walker metric:

$$
d s^{2}=a^{2}(\eta)\left(d \eta^{2}+2 S_{i} d \eta d x^{i}-\left(\delta_{i j}+2 F_{i, j}\right) d x^{i} d x^{j}\right)
$$

Notice that we are assuming a spatially-flat universe and accordingly for the unperturbed spatial metric we have $g_{i j}=-a^{2} \delta_{i j}$. The vector perturbations $S_{i}$ and $F_{i}$ are written in the notation of [24]. As commented above, for the dipole contribution we consider fluid velocities depending only on time, i.e. we will limit ourselves to the zero-mode equations, and therefore, we can take $S_{i}=S_{i}(\eta)$ and $F_{i, j}=0$. Accordingly, the total energy-momentum tensor reads:

$$
\begin{aligned}
T_{0}^{0} & =\sum_{\alpha} \rho_{\alpha} \\
T_{i}^{0} & =\sum_{\alpha}\left(\rho_{\alpha}+p_{\alpha}\right)\left(S_{i}-v_{i \alpha}\right) \\
T^{i}{ }_{0} & =\sum_{\alpha}\left(\rho_{\alpha}+p_{\alpha}\right) v_{\alpha}^{i} \\
T^{i}{ }_{j} & =-\sum_{\alpha} p_{\alpha} \delta^{i}{ }_{j}
\end{aligned}
$$

Notice that we are considering only the epoch after matter-radiation decoupling, assuming that dark energy is also decoupled and for that reason we will ignore possible energy and momentum transfer effects.

We now calculate the linearized Einstein equations using (3) and (44). The $\left({ }^{0}{ }_{0}\right)$ and $\left(\begin{array}{c}i \\ j\end{array}\right)$ components are trivial, whereas the $\left({ }^{0}{ }_{i}\right)$ and $\left(\begin{array}{c}i \\ 0\end{array}\right)$ yield the condition:

$$
S^{i}=\frac{\sum_{\alpha}\left(\rho_{\alpha}+p_{\alpha}\right) v_{\alpha}^{i}}{\sum_{\alpha}\left(\rho_{\alpha}+p_{\alpha}\right)}
$$

On the other hand, the energy conservation equations are trivially satisfied, whereas the total momentum conservation implies:

$$
\frac{d}{d \eta}\left(a^{4} \sum_{\alpha}\left(\rho_{\alpha}+p_{\alpha}\right)\left(S^{i}-v_{\alpha}^{i}\right)\right)=0
$$

which is compatible with (5). In General Relativity the combination $\left(\rho_{\alpha}+p_{\alpha}\right)$ appearing in (5) plays the role of inertial mass density of the corresponding fluid (see [25]), and accordingly $S^{i}$ can be understood as the cosmic center of mass velocity. Notice that a pure cosmological constant has no inertial mass density. Since matter-radiation decoupling takes place in the matter dominated era, the cosmic center of mass velocity is determined after decoupling by the motion of matter and dark energy, radiation playing essentially no role.

The momentum conservation equation for each fluid:

$$
\frac{d}{d \eta}\left(a^{4}\left(\rho_{\alpha}+p_{\alpha}\right)\left(S^{i}-v_{\alpha}^{i}\right)\right)=0
$$

implies that the corresponding velocity relative to the center of mass frame scales as: $\left|\vec{S}-\vec{v}_{\alpha}\right| \propto a^{3 w_{\alpha}-1}$, i.e. it is constant in the case of radiation and scales as $a^{-1}$ for matter. In the case of dark energy the scaling properties will depend on the particular model under consideration, as we will see below. Notice that for the zero modes, the equations above contain all the information about the evolution of the velocity perturbations. 


\section{Effects on the CMB dipole}

Once we know the form of the perturbed metric, we can calculate the effect of fluids motion on photons propagating from the last scattering surface using standard tools (see for instance [22] and references therein). The energy of a photon coming from direction $n^{\mu}=\left(1, n^{i}\right)$ with $\vec{n}^{2}=1$ as seen by an observer moving with velocity $u^{\mu}=a^{-1}\left(1, v^{i}\right)$ is given by:

$$
\mathcal{E}=g_{\mu \nu} u^{\mu} P^{\nu}
$$

with

$$
P^{\nu}=\frac{E}{a}\left(n^{\nu}+\frac{d \delta x^{\nu}}{d \eta}\right)
$$

where $E$ parametrizes the photon energy and the perturbed trajectory of the photon reads $x^{\mu}(\eta)=x_{0}^{\mu}(\eta)+\delta x^{\mu}$, with $x_{0}^{\mu}=n^{\mu} \eta$. To first order in the perturbation, assuming that the observer velocity is of the same order as the metric perturbation, we get:

$$
\mathcal{E} \simeq \frac{E}{a}\left(1+\frac{d \delta x^{0}}{d \eta}+\vec{n} \cdot(\vec{S}-\vec{v})\right)
$$

In order to obtain $d \delta x^{0} / d \eta$, we solve the geodesics equations to first order in the perturbations. In order to simplify the calculation, we notice that the geodesics corresponding to the $g_{\mu \nu}$ metric with affine parameter $\tau$ are the same as those corresponding to the $\hat{g}_{\mu \nu}=a^{-2} g_{\mu \nu}$ metric, with parameter $\eta$ such that $d \tau=a^{2} d \eta$. In such a case the unperturbed $\hat{g}_{\mu \nu}$ is nothing but the Minkowski metric, and the 0component of the geodesics equation reduces to:

$$
\frac{d^{2} \delta x^{0}}{d \eta^{2}}=0
$$

By defining $\hat{\mathcal{E}}=a \mathcal{E}$, the temperature fluctuation generated by the Sachs-Wolfe effect in this particularly simple case reads:

$$
\begin{aligned}
\left.\frac{\delta T}{T}\right|_{\text {dipole }} & =\left.\frac{\hat{\mathcal{E}}_{0}-\hat{\mathcal{E}}_{\text {dec }}}{\hat{\mathcal{E}}_{\text {dec }}} \simeq \frac{d \delta x^{0}}{d \eta}\right|_{\text {dec }} ^{0}+\left.\vec{n} \cdot(\vec{S}-\vec{v})\right|_{\text {dec }} ^{0} \\
& \left.\simeq \vec{n} \cdot(\vec{S}-\vec{v})\right|_{\text {dec }} ^{0}
\end{aligned}
$$

where the indices 0 , dec denote the present and decoupling times respectively, and we have made use of (11).

At decoupling, the universe is matter dominated and it is a good approximation

to neglect the contribution to $\vec{S}$ from dark energy. Since baryons and radiation were coupled until recombination, we take the velocity of matter $\vec{v}_{M}^{\text {dec }}$ to be the same as that of radiation at that time $\vec{v}_{R}^{d e c}$, and accordingly we have $\vec{S}_{d e c} \simeq \vec{v}_{M}^{d e c} \simeq \vec{v}_{R}^{\text {dec }}$. Here we are assuming for simplicity that baryonic and dark matter share a common rest frame. On the other hand, if we assume that the intrinsic density fluctuations in the last scattering surface contribute only a small fraction to the CMB dipole, it is a good approximation to take the emitter velocity to be $\vec{v}_{d e c} \simeq \vec{v}_{M}^{d e c}$. 
On the other hand, today the contribution of radiation to the energy density is negligible and from (5) we get:

$$
\vec{S}_{0} \simeq \frac{\Omega_{M} \vec{v}_{M}^{0}+\left(1+w_{\Lambda}^{0}\right) \Omega_{\Lambda} \vec{v}_{\Lambda}^{0}}{1+w_{\Lambda}^{0} \Omega_{\Lambda}}
$$

so that we find:

$$
\begin{aligned}
\left.\frac{\delta T}{T}\right|_{\text {dipole }} & \simeq \vec{n} \cdot\left(\vec{S}_{0}-\vec{v}_{0}\right) \\
& \simeq \vec{n} \cdot \frac{\Omega_{M}\left(\vec{v}_{M}^{0}-\vec{v}_{0}\right)+\left(1+w_{\Lambda}^{0}\right) \Omega_{\Lambda}\left(\vec{v}_{\Lambda}^{0}-\vec{v}_{0}\right)}{1+w_{\Lambda}^{0} \Omega_{\Lambda}}
\end{aligned}
$$

where $w_{\Lambda}^{0}=w_{\Lambda}(0)$ is the present value of the dark energy equation of state and we have used $\Omega_{M}+\Omega_{\Lambda}=1$.

According to this result, the $\mathrm{CMB}$ dipole is due to the relative velocity of the observer with respect to the present cosmic center of mass. In the particular case in which matter, radiation and dark energy share a common rest frame, i.e. $\vec{v}_{M}^{0}=\vec{v}_{R}^{0}=\vec{v}_{\Lambda}^{0}$ then the previous result reduces to the usual expression for the dipole: $\delta T /\left.T\right|_{\text {dipole }} \simeq$ $\vec{n} \cdot\left(\vec{v}_{R}^{0}-\vec{v}_{0}\right)$. However this needs not to be necessarily the case. Thus, in particular, it is possible that an observer at rest with radiation $\vec{v}_{0}=\vec{v}_{R}^{0} \neq \vec{v}_{M}^{0} \neq \vec{v}_{\Lambda}^{0}$ can measure an nonvanishing dipole according to (14).

Although in Standard Cosmology we expect $\vec{v}_{R}^{0}=\vec{v}_{M}^{0}$, provided matter and radiation shared a common rest frame until decoupling, we have seen that the existence of large scale bulk flows would suggest this not to be the case. There are indeed proposals in the literature in which matter and radiation rest frames started to differ after recombination [12]. For that reason, in this work, we have allowed for possible velocity differences today.

\section{Matter bulk flows and moving dark energy}

In the absence of dark energy or in the case in which it is in the form of a pure cosmological constant $\left(w_{\Lambda}=-1\right)$, dark energy would not contribute to the center of mass motion. Moreover, today the radiation contribution is negligible and accordingly the center of mass rest frame would coincide with the matter rest frame. As commented above, there are works in which matter and radiation rest frames start to differ after decoupling, and such an offset has been claimed to generate bulk flows on large scales. However our results imply that the relative motion of matter and radiation today could not explain the existence of bulk flows on the largest scales, since the frame in which the dipole vanishes would coincide with the matter rest frame. Conversely, the existence of non-vanishing bulk flows would require the presence of moving dark energy with $w_{\Lambda}^{0} \neq-1$.

Indeed, if moving dark energy is responsible for the existence of cosmic bulk flows on very large scales, then the amplitude and direction of such flows would provide a direct measurement of the relative velocity of matter and dark energy. The bulk flow 
$\vec{V}_{b}$ can be understood as the average velocity of a given matter volume with respect to an observer who measures a vanishing CMB dipole, i.e. $\vec{V}_{b}=\vec{v}_{M}^{0}-\vec{v}_{0}$. Such an observer has a velocity which is given, according to (14), by:

$$
\vec{v}_{0} \simeq \vec{v}_{M}^{0}+\frac{\left(1+w_{\Lambda}^{0}\right) \Omega_{\Lambda}}{1+w_{\Lambda}^{0} \Omega_{\Lambda}}\left(\vec{v}_{\Lambda}^{0}-\vec{v}_{M}^{0}\right)
$$

so that

$$
\vec{v}_{M}^{0}-\vec{v}_{\Lambda}^{0} \simeq \frac{1+w_{\Lambda}^{0} \Omega_{\Lambda}}{\left(1+w_{\Lambda}^{0}\right) \Omega_{\Lambda}} \vec{V}_{b}=P_{\Lambda} \vec{V}_{b}
$$

Notice that curiously, according to these results, even if matter is at rest with respect to the CMB radiation, $\vec{v}_{M}^{0}=\vec{v}_{R}^{0}$, it would be possible to have a non-vanishing flow $\vec{V}_{b} \neq 0$, provided dark energy is moving with respect to matter. The proportionality constant $P_{\Lambda}$ depends on the present value of $w_{\Lambda}$. Thus for instance, taking $\Omega_{\Lambda}=0.73$, we get $P_{\Lambda}=2.68$ for $w_{\Lambda}^{0}=-0.78$ or $P_{\Lambda}=14$ for the central values of the WMAP three-year data $\Omega_{\Lambda}=0.72$ and $w_{\Lambda}^{0}=-0.97$.

\section{Discussion}

Notice that bulk velocities of several hundred $\mathrm{km} \mathrm{s}^{-1}$ are obtained in some recent large scale bulk flow measurements. Therefore, in order for moving dark energy to have measurable effects, the relative matter-dark energy relative velocity today should be in the range $\gtrsim 10^{2} \mathrm{~km} \mathrm{~s}^{-1}$. Since the nature of dark energy is still unknown, and there is not a generally accepted model, we can only limit ourselves to show that those relative velocities are not a priori excluded, and that, in fact, there is a wide class of models in which such values can be obtained in a natural way.

As implied by (7), the relative velocity of dark energy with respect to the center of mass frame scales as $\left|\vec{S}-\vec{v}_{\Lambda}\right| \propto a^{3 w_{\Lambda}(z)-1}$. Thus, in models with constant equation of state, the relative velocity decreases faster than $a^{-3.3}$, since as commented before $w_{\Lambda}<-0.78$, so that any initial relative velocity is rapidly damped away. However there is another class of models in which the dark energy equation of state exhibits scaling behavior, i.e., it mimics that of the dominant component of the universe during most of the cosmological evolution. Such models have in addition the interesting property of being able to alleviate the fine tuning problem of models with constant equation of state. Examples of scaling models include quintessence [19], k-essence [20] and other phenomenological proposals (see [26] and references therein). In such models $w_{\Lambda}(z) \simeq 1 / 3$ for $z \gg z_{s}, w_{\Lambda}(z) \simeq 0$ for $z_{b} \ll z \ll z_{s}$ and $w_{\Lambda}(z) \simeq-1$ for $z \ll z_{b}$ $\left(z_{s} \sim \mathcal{O}\left(10^{3}\right)\right.$ and $z_{b} \sim \mathcal{O}(1)$ being typical values [26]). This means that, according to the above scaling behavior, no damping of the relative velocity would have taken place until $z \sim z_{s}$. Then a mild damping $\sim a^{-1}$ would have occurred for $z_{b} \lesssim z \lesssim z_{s}$, and only very recently $\left(z \lesssim z_{b}\right)$, the damping would have been stronger $\sim a^{-4}$, i.e. we expect typical total damping factors today around $10^{-3}-10^{-4}$ (depending on the exact transition redshifts). If the nature of dark energy is really independent of the rest of components of the universe, and it has been always decoupled from them, then the corresponding 
initial dark energy bulk velocity should be considered as a free cosmological parameter. Accordingly the present relative velocity would be comparable to the amplitudes of the observed CMB dipole and bulk flows measurements for initial values around $\left|\vec{S}-\vec{v}_{\Lambda}\right| \lesssim 1$.

A detailed analysis of the different dark energy models will be presented elsewhere 27. In any case, regardless the particular mechanism responsible for the motion of dark energy, it is interesting to note, according to the previous discussion, that a better determination of the existence of matter flows on very large scales could shed light on the nature of dark energy.

Finally, we would like to mention that the metric anisotropies created by the relative motion of fluids 28] could also have observable effects on photons and other types of particles propagating from sources located at cosmological distances [29]. Such effects could offer independent evidence of the motion of dark energy [27].

Acknowledgments: This work has been partially supported by DGICYT (Spain) under project numbers FPA 2004-02602 and FPA 2005-02327

\section{References}

[1] A.G. Riess, M. Davis, J. Backer and R.P. Kirshner, Astrophys. J. 488, L1 (1997); D.A. Dale et al. Astrophys.J. 510, L11 (1999); M. Colles et al., MNRAS, 321, 277 (2001)

[2] T.R. Lauer and M. Postman, Astrophys.J. 425, 418 (1994)

[3] D. D. Kocevski and H. Ebeling, astro-ph/0510106 P. Erdogdu et al., astro-ph/0507166

[4] M.J. Hudson et al. Astrophys.J. 512, L79 (1999); J.A. Willick, Astrophys.J. 522, 647 (1999)

[5] M.J. Hudson, R.J. Smith, J.R. Lucey and E. Branchini, Mon. Not. Roy. Astron. Soc. 352, 61 (2004); M.J. Hudson, astro-ph/0311072

[6] M.A. Strauss, R. Cen, J.P. Ostriker, T.R. Lauer and M. Postman, Astrophys.J. 444, 507 (1995)

[7] P.J.E. Peebles and D.T. Wilkinson, Phys. Rev. 174, 2168 (1968); P.J.E. Peebles, Principles of Physical Cosmology, Princeton University Press (1993)

[8] T. Padmanabhan, Structure formation in the universe, Cambridge University Press, (1993)

[9] A. Kogut et al., Astrophys.J. 419, 1 (1993); C.L. Bennett et al. Astrophys.J.Suppl. 148, 1 (2003)

[10] A. Yahil, G.A. Tammann and A. Sandage, Astrophys. J. 217, 903 (1977); S. Courteau and S. Van den Bergh, Astron. J. 118, 337 (1999)

[11] A.G. Riess, W.H. Press and R.P. Kirshner, Astrophys. J. 445, L91 (1995)

[12] R.A. Matzner, Astrophys. J. 241, 851 (1980)

[13] B. Paczynski and T. Piran, Astrophys. J. 364, 341 (1990)

[14] D. Langlois and T. Piran, Phys.Rev. D53, 2908 (1996)

[15] M.S. Turner, Phys. Rev. D44, 3737 (1991)

[16] S. Perlmutter et al., Astrophys. J. 517, 565 (1999); A.G. Riess et al., Astrophys. J., 607, 665 (2004)

[17] D.N. Spergel et al., Astrophys. J. Suppl. 148, 175 (2003)

[18] D.N. Spergel et al., astro-ph/0603449

[19] B. Ratra and P.J.E. Peebles, Phys. Rev. D37, 3406 (1988); C. Wetterich, Nucl. Phys. B302, 668 (1988); R.R. Caldwell, R. Dave and P.J. Steinhardt, Phys. Rev. Lett. 80, 1582 (1998)

[20] C. Armendariz-Picon, T. Damour and V. Mukhanov, Phys. Lett. B458, 209 (1999); T. Chiba, T. Okabe and M. Yamaguchi, Phys. Rev. D62, 023511 (2000)

[21] P.J.E. Peebles and B. Ratra, Rev. Mod. Phys. 75, 559, (2003)

[22] M. Giovannini, Int. J. Mod. Phys. D14, 363, (2005) 
[23] T. Moroi and T. Takahashi, Phys. Rev. Lett. 92, 091301, (2004); C. Gordon and W. Hu, Phys. Rev. D70, 083003, (2004)

[24] V.F. Mukhanov, H.A. Feldman and R.H. Brandenberger, Phys. Rep. 215 203, (1992)

[25] B. Schutz, A first course in general relativity, Cambridge University Press, (1985)

[26] S. Capozziello, A. Melchiorri and A. Schirone, Phys. Rev. D70, 101301 (2004)

[27] A.L. Maroto, work in preparation

[28] P.S. Letelier, Phys. Rev. D22, 807 (1980); S.S. Bayin, Astrophys. J. 303, 101 (1986)

[29] V.A. Kostelecky and M. Mewes, Phys. Rev. D66, 056005 (2002) 